\title{
Attenuated vaccines for tropical theileriosis, babesiosis and heartwater: the continuing necessity
}

\author{
Varda Shkap ${ }^{1}$, Albertus J. de Vos $^{2}$, Erich Zweygarth ${ }^{3}$ and Frans Jongejan ${ }^{4,5}$ \\ ${ }^{1}$ Division of Parasitology, Kimron Veterinary Institute, Bet Dagan, Israel \\ ${ }^{2}$ Tick Fever Centre, Department of Primary Industries, 280 Grindle Road, Wacol, Queensland 4076, Australia \\ ${ }^{3}$ Onderstepoort Veterinary Institute, Private Bag X5, Onderstepoort 0110, South Africa \\ ${ }^{4}$ Utrecht Centre for Tick-borne Diseases (UCTD), Department of Infectious Diseases and Immunology, Faculty of Veterinary \\ Medicine, Utrecht University, Yalelaan 1, 3584 CL, Utrecht, The Netherlands \\ ${ }^{5}$ Department of Veterinary Tropical Diseases, Faculty of Veterinary Science, University of Pretoria, Private Bag X04, 0110 , \\ Onderstepoort, South Africa
}

\begin{abstract}
Overwhelming evidence has accumulated of the effectiveness of immunization with live attenuated vaccines to control tick-borne diseases of livestock. Despite several disadvantages, vaccination with live attenuated organisms against tropical theileriosis, babesiosis and possibly heartwater constitutes one of the most cost-effective intervention strategies. Although great advances have been made through genomics and proteomics research, this has not yet translated into effective non-living vaccines. As a result, there is a continuing necessity to use available live vaccines in tick and tick-borne disease-control strategies adapted to conditions prevailing in many parts of the world.
\end{abstract}

\section{The need for vaccines}

Tick-borne diseases constitute a major constraint of livestock production and have considerable economic impact [1]. In general, tick-borne protozoan diseases (e.g. theileriosis and babesiosis) and rickettsial diseases (e.g. heartwater or cowdriosis and anaplasmosis) are major health and management problems of cattle and small ruminants in Africa, Australia, Asia and Latin America. In cattle, tropical theileriosis and babesiosis are caused by protozoan parasites (e.g. Theileria annulata, Babesia bovis and Babesia bigemina), whereas heartwater and anaplasmosis are caused by rickettsias, Ehrlichia ruminantium and Anaplasma marginale, respectively. In endemic areas, indigenous cattle have developed resistance to ticks and tick-borne pathogens [1]. However, susceptibility of exotic breeds presents a major obstacle to the improvement of cattle production. Chemical tick control, treatment of animals, chemoprophylaxis and vaccination are among the measures available to limit losses incurred by tick-borne diseases. However, chemical control is limited owing to selection of acaricide-resistant ticks; in addition, residues in meat and milk have raised public health concerns. Intensive acaricide

Corresponding author: Shkap, V. (shkapv@int.gov.il).

Available online 25 July 2007. practice also interferes with enzootic stability, rendering animals susceptible to the diseases. Chemoprophylaxis can be effective, but for a short period only. As a result, sustainable control has been based on vaccination using live attenuated organisms. Here, we focus on attenuated vaccines against tropical theileriosis, babesiosis and heartwater. Attenuation of $A$. marginale has not been achieved, and no $A$. marginale live attenuated vaccine is available [2]. The Anaplasma centrale successfully used for vaccination in Australia, South Africa, several countries in Latin America and Israel, was not attenuated but, instead, isolated as an organism of naturally low pathogenicity. The live vaccines induce a long-lasting immunity but their use has disadvantages; therefore, development of alternative approaches is necessary. Research in the post-genomic era will undoubtedly lead to novel genomic-based approaches to the development of recombinant vaccines $[3,4]$ but, in the meantime, we need to make use of existing methods of immunization.

\section{Pros and cons of different types of vaccines}

The use of live, blood-derived vaccines entails some problems, including: (i) the possible spread of silent pathogens such as bovine leukemia virus; (ii) difficulties in standardizing the vaccine dose; (iii) the risk of reversion of virulence; (iv) maintenance of carrier animals, which might serve as reservoirs for tick transmission; and (v) quality control of vaccine production, maintenance and transportation to the end user, including the necessity for a cold chain. For production of a culture-derived vaccine against theileriosis, commercially available and quality-assured reagents are used; therefore, the risk for spreading pathogens is minimal. For Babesia cultures, supply of fresh bovine erythrocytes and serum from specific donors is required, and maintenance of these donors requires highly stringent conditions. Using crude and soluble Babesia antigens would overcome the difficulties inherent in production of live anti-Babesia vaccines $[5,6]$, but immunity against heterologous challenge imparted by these vaccines have not been well documented, with the possible exception of Babesia 
divergens [7]. Success has been reported for soluble parasite antigens from Babesia canis and Babesia rossi in vitro cultured parasites, which are used for vaccination of dogs [8]. For heartwater, the only available vaccine is based on live organisms derived from the blood of infected sheep, and its use is limited to South Africa [9]. The vaccine is administered intravenously and, following inoculation, body temperature is monitored and antibiotic treatment has to be applied. In fact, this procedure is not a vaccination, but an infection-and-treatment method [10]. Importantly, the specific feature of the Ball 3 stock is that the temperature rise takes place several days before clinical disease occurs. Although other stocks might provide better cross-protection against field strains, they might be fatal shortly after a rapid temperature rise, by which time treatment is no longer effective. Therefore, the more virulent stocks are not suitable for prophylactic use in the infection-and-treatment method, whereas, for the past 50 years, the effectiveness of the Ball 3 stock against heartwater has proved satisfactory, but by no means complete.

\section{Attenuated vaccine against tropical theileriosis}

$T$. annulata, which causes tropical theileriosis, is transmitted by Hyalomma ticks. Clinical signs are fever, enlarged lymph nodes, anemia, icterus and haemoglobinuria. The disease causes up to $\sim 30 \%$ mortality, varying between regions and breeds of cattle [11]. The life cycle [12] involves three developmental stages: sporozoites, schizonts and piroplasms. Sporozoites, which are transmitted by Hyalomma ticks, rapidly invade monocytes and macrophages, wherein they transform into schizonts (multinucleated parasites), which induce proliferation of the host cell. The schizonts are divided over the two daughter cells upon host-cell division (Figure 1a,b), and the merozoites, which are formed in the schizonts, are released when infected cells rupture and rapidly invade erythrocytes. Inside the red blood cell, they develop into the piroplasm stage [13] (Figure 1c). Of all stages, only schizonts can be grown in vitro and, after prolonged cultivation, they become attenuated, which is the basis for culture-derived vaccines [14].

Cattle that have recovered after sporozoite challenge are protected against all other stages. Following infection with schizonts, which are responsible for the main pathology, protective immunity is induced against homologous challenge, whereas partial immunity is induced against heterologous challenge or challenge by infected ticks [14].
Piroplasms found in persistently infected cattle do not protect against infection with schizonts or sporozoites [15]. By contrast, immunity induced by schizonts has little effect on the production of piroplasms developed upon natural infection. The attenuated cell-culture-derived vaccine against tropical theileriosis has proved to be safe for all breeds of cattle. The degree of protection varies with age and breed of cattle. Dairy cattle are more sensitive to T. annulata, and, following tick infection might respond with fever and parasitaemia. Upon complete attenuation, no piroplasms can be detected in blood, which implies that the vaccine does not provide a reservoir of infection for ticks. However, because vaccination does not prevent development of piroplasms following tick transmission, eradication of the disease cannot be achieved by immunization [16]. Vaccination during an ongoing outbreak does not aggravate the outcome of the disease; on the contrary, it prevents additional clinical cases. Using more sensitive techniques, such as reverse blot hybridization, piroplasms were detected in calves from Spain and Sudan following immunization with attenuated schizont-infected cells. Despite this, transmission of the carrier-status infection by Hyalomma ticks failed, which indicates a low risk for spreading the vaccine strains in the field [17]. It is important to emphasize that reversion to virulence has not been reported after the schizonts are completely attenuated. The vaccine induces immunity that lasts from 14 months to four years $[18,19]$ but revaccination might be required if cattle are not exposed to natural challenge. Field isolates present a mix of antigenically diverse parasite populations, and accumulated evidence has shown that this has a limited impact on the protective immunity induced by the vaccine [14]. Analysis of parasite-population structures based on the major merozoite and piroplasm surface protein Tams1, has provided additional evidence for the broad protection engendered by attenuated cell-culture vaccines against heterologous field challenge [20].

\section{Mechanisms of attenuation}

Mechanisms of attenuation have yet to be elucidated. Attenuation is likely to be multifactorial, involving selection of avirulent parasite subpopulations and alterations in gene expression [21]. Phenotypic and genotypic alterations in gene expression have been shown by the loss of expression of parasite-induced matrix metalloproteases during attenuation, and the inability to produce merozoites was shown by differential RNA display [21-23].

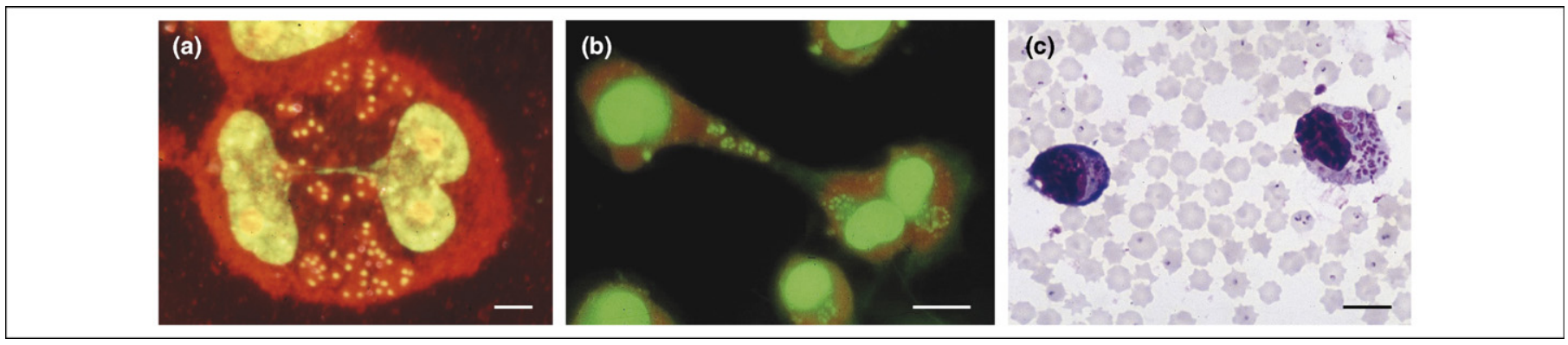

Figure 1. Developmental stages of Theileria annulata in cell culture and peripheral blood of infected calf. (a) $T$. annulata schizont scattered in the cytoplasm of an infected cell. T. annulata schizont divided together with the host cell (acridine orange staining). (c) T. annulata schizont and piroplasms in a blood smear (Giemsa staining). Scale bars $=10 \mu \mathrm{m}$. 


\section{Protective immune mechanisms}

The mechanism of protective immunity is mainly cell-mediated; it involves both innate and adaptive immune responses, and is directed against the schizont stage [12]. In vivo, T. annulata preferentially infects monocytes and macrophages rather than lymphoid cells [24]. It has been suggested that infected cells function as antigen-presenting cells, which stimulate antigen-specific memory T cells [25]. Importantly, the vaccine is effective when attenuated schizonts are transferred from donor to recipient cells, where they establish themselves and transform the host cell [16].

The innate immune response involves activation of macrophages, suppressing proliferation of infected cells, production of tumor necrosis factor $\alpha(\mathrm{TNF}-\alpha)$, nitric oxide and natural killer cells [12]. The adaptive response mounts $\mathrm{T}$ cells that are cytotoxic for schizont-infected cells, antigensensitized lymphocytes and a T-helper cell-type $1 \mathrm{CD} 4^{+}$ response: production of TNF- $\alpha$ and IFN- $\gamma$, with 'cytostatic' macrophages producing nitric oxide, the effective mediator of the protective response to schizont-infected cells in vivo $[12,24]$. Interestingly, the same cytokines are also implied in pathology. Thus, activity of pro-inflammatory cytokines predetermines either immunity or pathology [25]. In cattle infected with sporozoites the response is rapid with innate mechanisms operating within $24 \mathrm{~h}$, whereas cattle inoculated with schizonts respond only after the schizonts have developed in the recipient host cells. Therefore, the speed of the innate response and the rapid recall of the $\mathrm{CD} 4^{+} \mathrm{T}$-cell response are considered crucial in determining the efficacy of protective immunity [12].

Recently, immunization of cattle with sporozoite surface antigen 1 or attenuated schizont-infected cells induced limited protection against homologous or heterologous sporozoite challenge, whereas a combination of recombinant and live vaccine resulted in survival of all vaccinates [26]. These results provide evidence for achieving improved protective immunity by inclusion of sporozoite and schizont antigens in new generation vaccines to be developed.

\section{Implementation of the vaccine}

Tropical theileriosis occurs in the Mediterranean coastal regions (Portugal, Spain, Italy, Greece, Turkey, Israel), northern Africa, Mauritania and surroundings of the Nile River in northern Sudan, the Middle East, southern Caucasus Mountains, extending to Pakistan, India, Afghanistan, Uzbekistan, Kazakhstan and north-eastern China. However, the actual area where attenuated Theileria vaccines have made impact is much smaller because vaccines have been implemented only in Israel, Iran, Morocco, Tunisia, India, China and Uzbekistan. Several requirements need to be in place to ensure production and implementation of the vaccine [27] (Box 1). Support from veterinary authorities to implement production and application is considered to be crucial in promoting vaccination on a national basis.

\section{Attenuated vaccines against babesiosis}

$B$. bovis and B. bigemina are economically important parasites of cattle that are transmitted by Boophilus ticks [28] and widespread in Asia, Latin America and Africa. These intra-erythrocytic parasites cause destruction of host cells, which results in fever, haemoglobinuria and
Box 1. Essential requirements for production and implementation of Theileria annulata vaccine [29]

- Isolation of schizonts at an acute stage of the disease.

- Initiation of schizont-infected cell culture from buffy coat, lymphnode biopsy material, or trypsinized lymph node, spleen or liver post-mortem material.

- Attenuation of virulence by continuous sub-cultivation of infected cells.

- Inoculation of $5 \times 10^{6}$ infected cells from every 20th passage into each of two susceptible calves to evaluate attenuation of virulence.

- Freezing and storage of every $10-20$ passage in liquid nitrogen (or $\left.-70{ }^{\circ} \mathrm{C}\right)$.

- Evaluation of attenuation: schizont-infected cells are considered attenuated when neither fever nor schizonts in lymph node or liver biopsy, or piroplasms are detected in Giemsa-stained smears upon inoculation.

- Preparation of frozen master-, working- and production-seed materials for mass culture production.

- Production of frozen concentrated $10 \times$ or $20 \times$ vaccine, with $1 \times 10^{7}$ per dose of infected cells cryo-preserved with $7 \%$ dimethyl sulfoxide (DMSO).

- Vaccine post-production testing for sterility.

- Vaccine post-production testing for safety (inoculation of susceptible calves with 10x greater doses) and efficacy (challenge of immunized and control calves 6-8 weeks after vaccination with a sporozoite stabilate, containing the equivalent of up to five infected Hyalomma spp. ticks).

- Testing for viability of vaccine thawed cells by dye-exclusion assay, or examination of culture-plating efficiency in vitro.

- Storage and transportation of the vaccine to farms in liquid nitrogen.

- Handling of frozen vaccine: upon thawing to $37^{\circ} \mathrm{C}$, the content of the tube with the concentrated infected cells is transferred into a bottle of diluent, containing $7 \%$ DMSO in phosphate buffered saline (PBS), and inoculation required within $1 \mathrm{~h}$.

anemia, and, in the case of $B$. bovis infection, fatal cerebral babesiosis associated with sequestration of infected erythrocytes in capillaries of the brain (Figure 2a-d). Mortality rates are high in susceptible European cattle breeds. Prophylactic immunization has proved most effective to reduce losses caused by babesiosis. Vaccines incorporating live attenuated strains of $B$. bovis and B. bigemina have been used for many years, in particular, in Australia, Argentina, Brazil, Uruguay, South Africa and Israel. There is capability to produce these vaccines in Colombia, Sri Lanka, Zimbabwe and Malawi. Splenectomized calves are the most popular source of immunogens [29,30], although parasites grown in vitro are used with great success in Argentina [31]. For dispatch, the vaccine is chilled or cryopreserved, and the viability of the parasites can be determined with the calcein stain (Figure 2d). Disadvantages of blood-derived live immunogens are well known; they include short shelf life (for chilled vaccine), risks of vaccine reactions or contamination and reversion to virulence $[5,32]$. There is also a potential loss of immunogenicity after long-term maintenance in vitro [5]. To counter these deficiencies, standardized production methods have been developed [29,33] (Box 2).

\section{Protective immune mechanisms}

Field isolates of $B$. bovis comprise a range of subpopulations that express virulence genes to varying degrees [34]. Attenuation by passage in splenectomized calves or maintenance 


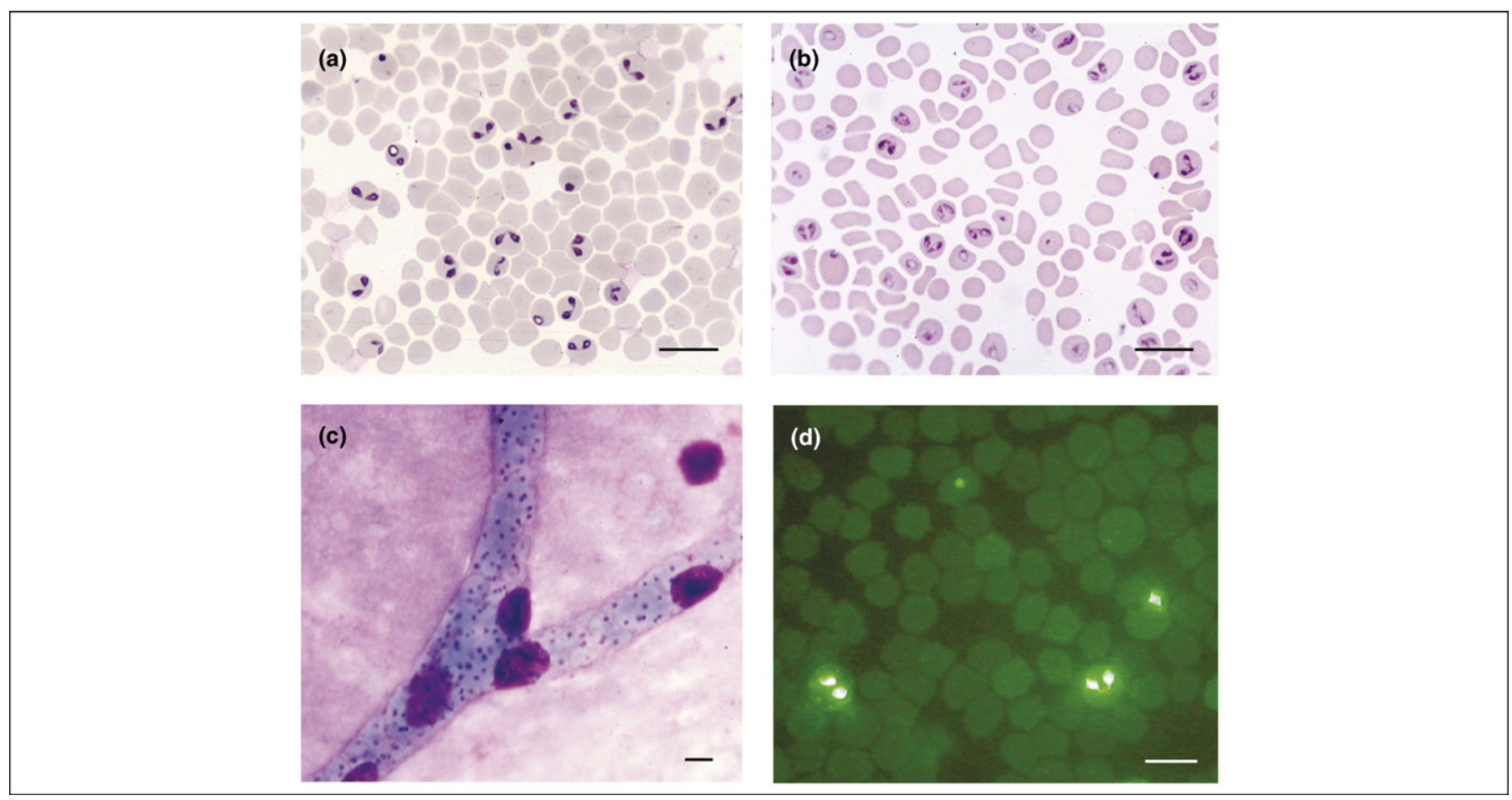

Figure 2. Blood smears from (a) Babesia bovis and (b) Babesia bigemina. (c) B. bovis in a brain capillary smear (Giemsa staining), (d) B. bovis (vitality staining with calcein), Scale bars $=10 \mu \mathrm{m}$.

in culture can result in enrichment of less virulent parasite subpopulations or in down-regulation of virulence genes [5]. Protective immunity is unrelated to virulence of the vaccine strain and there is evidence that multiple passage of a strain might result in loss of immunogenicity [35]. Consequently, some vaccine strains become less immunogenic than others, and there is evidence that a combination of differing parasite populations is required for adequate protection. At present, the vaccine strain used in Australia comprises two different parasite populations. Reversion to virulence following passage of an attenuated vaccine strain through ticks was reported for $B$. bovis [34].

The virulence of $B$. bigemina isolates decreases during prolonged residence in latently infected animals. This characteristic has been exploited to obtain avirulent strains by splenectomizing latently infected calves and using the resulting relapse parasites to repeat the procedure [36]. Both Babesia spp. are immunologically diverse, and analysis of DNA extracts of 27 Australian isolates of $B$. bovis using polymerase chain reaction (PCR) showed all 27 to be different [37]. Antigenic variation is thought to avoid strong protective responses mounted by the host [38]. Despite this immunological diversity, the Australian B. bovis and $B$. bigemina strains protect cattle in Africa, Latin America and Southeast Asia [35].

The mechanisms of immunity to Babesia require both innate and adaptive responses [39]. Calves are more resistant to disease than older cattle, and this age-linked resistance is thought to be the result of natural killer cell responses in the spleens of young animals [40]. The mechanism of acquired immunity seems to rely on the rapid activation of memory and effector $\mathrm{CD} 4^{+} \mathrm{T}$ helper cells that secrete IFN- $\gamma$, thereby activating phagocytic cells and enhancing antibody production by B cells [39-41].
From experience gained in Australia, immunity to $B$. bovis and $B$. bigemina persists for approximately four years [35]. Sterile immunity followed by chemosterilization in the case of $B$. bovis was more than three years, but cattle were fully susceptible to B. bigemina after 16 months [30]. After elimination of the infection, immunity seems to be related more to the degree of antigenic stimulation experienced by the host than to the presence of live parasites.

Development of recombinant subunit vaccines against Babesia spp. has been slow. Immunization with native or recombinant rhoptry-associated proteins (RAP-1), which are targeted immunogens for vaccine development of $B$. bovis and $B$ bigemina, reduced parasitemia in infected cattle $[40,42]$. Immunization with the $\mathrm{N}$-terminal region containing immuno-dominant T-cell epitopes of $B$. bovis imparted no protection, despite specific RAP-1 immunoglobulin $\mathrm{G}$ and $\mathrm{CD}^{+}{ }^{+} \mathrm{T}$-cell responses to challenge [41]. Because immuno-dominant antigens eliciting strong cellmediated immune responses failed to confer protective immunity, it has been suggested that identification of subdominant antigens might be important for an effective vaccine [39-42]. The annotation of the $B$. bovis genome sequence should enable effective antigens to be identified. A protective vaccine might need to include multiple antigens [39] and its formulation would require a better understanding of antigenic diversity, mechanisms used by Babesia to evade host immunity and the heterogeneity of the bovine major histocompatibility complex class II molecules [40,42-44].

\section{Attenuated vaccines against heartwater}

Heartwater (E. ruminantium infection) occurs throughout sub-Saharan Africa and on several islands in the Caribbean [10]. The disease is transmitted by Amblyomma ticks 
Box 2. Essential requirements for production and implementation of Babesia vaccines [31]

- Isolation of Babesia parasites through feeding of infected ticks on splenectomized animals, or through cloning in cell culture.

- Maintenance of pathogen-free cattle under tick-free conditions to ensure freedom from arthropod-borne and other endemic infections.

- Facilities for splenectomy.

- Attenuation of the virulence of Babesia bovis by 'rapid passages' through splenectomized calves at the acute stage of infection, or by 'slow passages' in splenectomized persistently infected calves for attenuation of Babesia bigemina.

- Testing for viral and bacterial contaminants of infected blood for stabilate preparation.

- Resources and technology to test donors for freedom of contaminants.

- Preparation of master- and working-seed stabilates stored in liquid nitrogen (or $-70{ }^{\circ} \mathrm{C}$ freezer).

- Resources and technology to monitor population dynamics in working seed.

- Quality-assurance procedures to monitor working-seed safety and efficacy.

- Sterile condition facilities for production of frozen or chilled vaccine.

- Production of vaccines from an infected splenectomized donor at parasitemia of $1 \times 10^{8} \mathrm{ml}^{-1}$ (additional passage in a donor calf might be required for $B$. bovis to obtain suitable parasitemia for vaccine production).

- If frozen, infected blood is mixed with cryo-protectant (glycerol or DMSO) and dispersed at $10 \times$ or more concentrated doses into sterile vials.

- Diluents containing PBS and cryo-protectant at concentration similar to that in frozen vaccine are supplied with the vaccine and are kept at ambient temperature.

- Post-production testing for sterility for viral and bacterial contaminants.

- Testing for safety and potency by cattle inoculation and clinical follow up.

- Production of chilled vaccines at suitable parasitemia from donorinfected blood dispensed into sterile vials.

[45], and affects cattle, small and wild ruminants, with mortality rates of $77 \%$ in sheep and goats, and $67 \%$ in cattle [46]. In some regions, the disease is of greater economic importance in small ruminants, whereas elsewhere it presents mostly a constraint for cattle production. The infection and treatment method in present use is based on virulent organisms and the stock used in South Africa (Ball 3) does not provide wide protection against virulent field isolates; there has been a continuous search for a better and safer vaccine to avoid drug treatment. A breakthrough was reported in 1991 when an isolate of heartwater originating from Senegal lost its virulence during passage in bovine endothelial cell cultures [47]. Suspensions of these cultures used for vaccination produced strong protection against homologous challenge in sheep and goats. However, under laboratory conditions, the attenuated Senegal stock did not provide efficient cross-protection against geographically distinct virulent stocks [48]. Similarly, field trials conducted in Senegal showed that immunity to this stock did not seem to protect satisfactorily against strains encountered in the field [49]. In Guadeloupe, the Gardel stock was attenuated in vitro after $>200$ passages (D. Martinez, $\mathrm{PhD}$ thesis, Utrecht University, 1997). Recently, the virulent Welgevonden stock of $E$. ruminantium was continuously propagated in a canine macrophage-monocyte cell line (DH82) [50]. Between passages 25 and 50, the organisms lost virulence for mice; these were solidly protected against a lethal challenge. Sheep inoculated with the attenuated culture material of passages 56 and 111 were protected against $10 \mathrm{LD}_{50}$ of virulent homologous challenge 30 days after immunization: none of the immunized animals required chemotherapy, whereas the control sheep were severely sick [51]. Interestingly, adult Amblyomma hebraeum ticks fed as nymphs on immunized sheep were able to acquire the attenuated stock and transmit it to a naïve sheep, which was protected against lethal homologous challenge [52]. By contrast, the attenuated Senegal and Gardel stocks were not tick-transmissible (D. Martinez, $\mathrm{PhD}$ thesis, Utrecht University, 1997). In areas where the virulent Welgevonden stock is present, however, tick transmissibility need not be a disadvantage because ticks infected with the attenuated organisms could subsequently immunize naïve animals. The attenuated Welgevonden stock was re-adapted to a bovine endothelial cell-culture line (Figure 3a,b). Upon immunization, sheep and goats did not develop disease; a transient rise in body temperature was the only symptom observed. Importantly, all sheep were fully protected against a lethal challenge with the virulent homologous stock, or with one of four heterologous stocks (Ball 3, Gardel, Mara 87/7 and Blaauwkrans). Inoculation at doses of $1.8 \times 10^{4}$ and $6.0 \times 10^{3}$ organisms injected into sheep and goats, respectively, were sufficient to trigger a robust immune response [52].

\section{Protective immune mechanisms}

The mechanisms of protection engendered by live E. ruminantium have not been elucidated. Immunity is considered to be cell-mediated [53] because serum antibodies play no part in the immune response. Specific antibodies are produced following infection; however, their
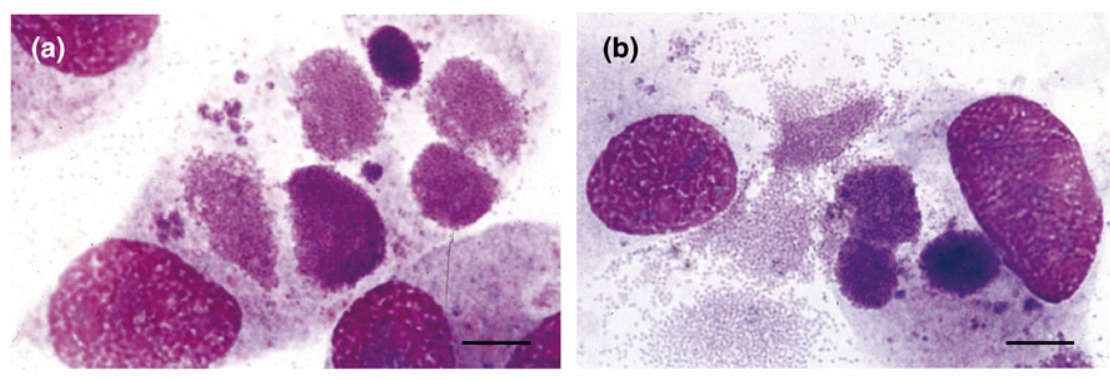

Figure 3. Bovine endothelial cells infected with Ehrlichia ruminantium: (a) six intact colonies in the cytoplasm; (b) three intact colonies and free elementary bodies released from the colonies (Giemsa staining). Scale bars $=10 \mu \mathrm{m}$. 
Box 3. Essential requirements for production and implementation of a culture-derived attenuated heartwater vaccine $^{*}$

- Isolation of the organism from infected ruminants or Amblyomma ticks.

- Adaptation of the isolate to in vitro propagation and continuous growth.

- Testing of cultured organisms for virulence at various passage levels in small ruminants.

- Titration of immunizing doses in small ruminants.

- Testing for potency against heterologous virulent challenge.

- Preparation of master-, working- and production-seed materials for mass culture production.

- Testing of the vaccine for contaminants.

- Availability of cold chain for production, maintenance and transportation of frozen vaccine.

E. Zweygarth, PhD Thesis, Utrecht University, 2006

level does not correlate with protection [54]. In cattle immunized by infection and treatment, the responses to recombinant analogs of major protein 1 (MAP1) and MAP2 were predominantly by $\mathrm{CD} 4^{+} \mathrm{T}$ cells. Cytokines INF- $\gamma$, INF- $\alpha$, TNF- $\beta$, interleukin-2-receptor $\alpha$ transcripts (IL$2 \mathrm{R} \alpha$ ) were strongly expressed with the application of reverse transcription PCR, whereas IL-2 and IL-4 were expressed weakly [55].

There are arguments in favor of using an attenuated vaccine against heartwater. The efficacy of the vaccine based on the Welgevonden stock has to be further evaluated in field trials. The possibility of a reversal of virulence will have to be studied and eliminated before it can be used outside South Africa to avoid introducing an additional genotype where it might not already occur. Most importantly, it confers cross-protection against most South African isolates [52,56], a blocking treatment with antibiotics is not required and it is less expensive to produce than the traditional blood vaccine. Efforts need to be undertaken to determine the immunogens that elicit protective cellmediated responses. An attenuated vaccine might be an ideal interim solution until a new-generation vaccine becomes available. The genome of $E$. ruminantium has been sequenced [4], making available information that will support a major contribution towards the development of a recombinant vaccine. For the present time, the requirements for the production of the viable anti-heartwater vaccine are given in Box 3.

\section{Concluding remarks}

Vaccination with live attenuated vaccines is an effective means of controlling tropical theileriosis and babesiosis in cattle. The attenuated vaccine against heartwater is still in the experimental stage, and wider field-testing is required in cattle and goats, and the age of animals to be vaccinated has not yet been determined. Vaccines for controlling $T$. annulata and $E$. ruminantium infection are produced from cell cultures; therefore, risks of contamination with viral or bacterial pathogens can be better controlled. The vaccine against tropical theileriosis is safe for all breeds of cattle at any age, and can even be used for pregnant cows. The blood-derived Babesia vaccines require stringent maintenance conditions and thorough examination of donor cattle. However, these vaccines confer the solid protective immunity against natural infection, which justifies their use for controlling these diseases.

\section{Future perspectives}

Development of technology for parasite transformation, gene knockout and gene silencing based on genomesequencing data will further contribute to a better understanding of the parasite biology and, ultimately, to a novel generation of vaccines. Nevertheless, in the immediate future, control of tropical theileriosis is likely to remain dependent on vaccination with a cell-culture-derived vaccine. Blood-based live attenuated anti-Babesia vaccines will continue to have a vital role in the near future; the immunogens propagated in vitro would be preferable but, for this to become the international standard, a better understanding of the long-term effects of in vitro maintenance of parasite immunogenicity is essential.

\section{Acknowledgements}

Writing of this article has been facilitated through The Integrated Consortium on Ticks and Tick-borne Diseases (ICTTD-3) financed by the International Cooperation Program of the European Union through Coordination Action Project no. 510561. We thank Gerrit Uilenberg and Alberto Guglielmone for helpful discussions.

\section{References}

1 Jongejan, F. and Uilenberg, G. (2004) The Global Importance of Ticks. Parasitology 129, S3-S14

2 Kocan, K. et al. (2003) Antigens and alternatives for control of Anaplasma marginale infection in cattle. Clin. Microbiol. Rev. 16, 698-712

3 Pain, A. et al. (2005) Genome of the host-cell transforming parasite Theileria annulata compared with T. parva. Science 309, 131-133

4 Collins, N. et al. (2005) The genome of the heartwater agent Ehrlichia ruminantium contains multiple tandem repeats of actively variable copy number. Proc. Natl. Acad. Sci. U. S. A. 102, 838-843

5 Bock, R. et al. (2004) Babesiosis of cattle. Parasitology 129, S247-S269

6 Rojas, C. et al. (2006) Bovine babesiosis live vaccine production. Ann. N. Y. Acad. Sci. 1081, 405-416

7 Edelhofer, R. et al. (1998) Improved disease resistance after Babesia divergens vaccination. Parasitol. Res. 84, 181-187

8 Schetters, T. et al. (2006) Onset and duration of immunity against Babesia canis infection in dogs vaccinated with antigens from culture supernatants. Vet. Parasitol. 138, 140-146

9 Bezuidenhout, J. (1989) Cowdria vaccines. In Veterinary Protozoan and Hemoparasite Vaccines (Wright, I.G., ed.), pp. 31-42, CRC Press

10 Uilenberg, G. (1983) Heartwater (Cowdria ruminantium infection): current status. Adv. Vet. Sci. Comp. Med. 27, 427-480

11 Luo, J. and Lu, W. (1997) Cattle theileriosis in China. Trop. Anim. Health Prod. 29 (Suppl. 4), 4S-7S

12 Preston, P. et al. (1999) Innate and adaptive immune responses cooperate to protect cattle against Theileria annulata. Parasitol. Today $15,268-274$

13 Shiels, B. (1999) Should I stay or should I go now: a stochastic model of stage differentiation in Theileria annulata. Parasitol. Today 15, 241-245

14 Pipano, E. and Shkap, V. (2000) Vaccination against tropical theileriosis. Ann. N. Y. Acad. Sci. 916, 484-500

15 Uilenberg, G. et al. (1986) Stage specific antigenicity in Theileria annulata: a case report. Vet. Q. 8, 73-75

16 Boulter, N. and Hall, R. (2000) Immunity and vaccine development in the bovine theilerioses. Adv. Parasitol. 44, 42-97

17 Gubbels, M. et al. (2000) Characterization of attenuated Theileria annulata vaccines from Spain and the Sudan. Ann. N. Y. Acad. Sci. 916, 521-532

18 Shkap, V. and Pipano, E. (2000) Culture-derived parasites in vaccination of cattle against tick-borne diseases. Ann. N. Y. Acad. Sci. $916,155-171$ 
19 Singh, S. et al. (2001) Field vaccination with a Theileria annulata schizont cell culture vaccine on the epidemiology of tropical theileriosis. Vet. Parasitol. 101, 91-1007

20 Gubbels, M. et al. (2001) Study of Theileria annulata population structure during bovine infection and following transmission to ticks. Parasitology 123, 553-561

21 Hall, R. et al. (1999) Mechanism(s) of attenuation of Theileria annulata vaccine cell lines. Trop. Med. Int. Health 4, A78-A84

22 Sommerville, R. et al. (1998) Phenotypic and genomic alterations underlying the attenuation of a Theileria annulata vaccine cell line from Turkey. Vaccine 16, 569-575

23 Shkap, V. et al. (2003) Proteolytic enzyme activity and attenuation of virulence in Theileria annulata schizont-infected cells. Vet. Parasitol. $115,247-255$

24 Forsyth, L. et al. (1997) Bovine cells infected in vivo with Theileria annulata expresses CD11b, the C3bi complement receptor. Vet. Res. Commun. 21, 249-263

25 Glass, E. (2001) The balance between protective immunity and pathogenesis in tropical theileriosis: what we need to know to design effective vaccines for the future. Res. Vet. Sci. 70, 71-75

26 Darghouth, M. et al. (2006) Vaccination of calves with attenuated cell line of Theileria annulata and the sporozoite antigen SPAG-1 produces a synergistic effect. Vet. Parasitol. 142, 54-62

27 Pipano, E. et al. (2004) Theileriosis. In Manual of Standards for Diagnostic Tests and Vaccines for Terrestrial Animals, pp. 523-536, OIE (http://www.oie.int/eng/normes/mmanual/A_00059.htm)

28 Brown, W. and Palmer, G. (1999) Designing blood-stage vaccines against Babesia bovis and B. bigemina. Parasitol. Today 15, 275-281

29 Bock, R. et al. (2004) Bovine babesiosis. In Manual of Standards for Diagnostic Tests and Vaccines for Terrestrial Animals, pp. 507-518, OIE (http://www.oie.int/eng/normes/mmanual/A_00059.htm)

30 De Waal, D. and Combrink, M. (2006) Live vaccines against bovine babesiosis. Vet. Parasitol. 138, 88-96

31 Mangold, A. et al. (1996) Viability after thawing and dilution of simultaneously cryopreserved vaccinal Babesia bovis and Babesia bigemina strains cultured in vitro. Vet. Parasitol. 61,345-348

32 Hope, M.et al. (2005) Potential for recombinant Babesia bovis antigens to protect against a highly virulent isolate. Parasite Immunol. 27, 439-445

33 De Vos, A. et al. (1997) Protozoal and rickettsial vaccines. In Vaccine Manual - The Production and Quality Control of Veterinary Vaccines for Use in Developing Countries (Mowat, G. and Rweyemamu, M., eds), pp. 75-89, FAO

34 Timms, P. et al. (1990) Study of virulence and vector transmission of Babesia bovis by use of cloned parasite lines. Infect. Immun. 58, 21712176

35 Bock, R. and de Vos, A.J. (2001) Immunity following use of Australian tick fever vaccine: a review of the evidence. Aust. Vet. J. 79, 832-839

36 Dalgliesh, R. et al. (1981) Development of a highly infective Babesia bigemina vaccine of reduced virulence. Aust. Vet. J. 57, 8-11

37 Lew, A. et al. (1997) Genotypic diversity in field isolates of Babesia bovis from cattle with babesiosis after vaccination. Aust. Vet. J. 75, 575-578

38 Allred, D. (2001) Antigenic variation in babesiosis: is there more than one 'why'? Microbes Infect. 3, 481-491
39 Brown, W.C. et al. (2006) Immune control of Babesia bovis infection. Vet. Parasitol. 138, 75-87

40 Brown, W.C. et al. (2006) Prospects for recombinant vaccines against Babesia bovis and related parasites. Parasite Immunol. 28, 315-327

41 Norimine, J. et al. (2003) Stimulation of T-helper cell $\gamma$ interferon and immunoglobulin G responses specific for Babesia bovis rhoptryassociated protein 1 (RAP-1) or a RAP-1 protein lacking the carboxy-terminal repeat region is insufficient to provide protective immunity against virulent $B$. bovis challenge. Infect. Immun. 79, 5021-5032

42 Wright, I.G. et al. (1992) The development of a recombinant Babesia vaccine. Vet. Parasitol. 44, 3-13

43 Carcy, B. et al. (2006) Genetic basis for GPI-anchor merozoite surface antigen polymorphism of Babesia and resulting antigenic diversity. Vet. Parasitol. 138, 33-49

44 Allred, D.R. and Al-Khedery, B. (2004) Antigenic variation and cytoadhesion in Babesia bovis and Plasmodium falciparum: different logics achieve the same goal. Mol. Biochem. Parasitol. 134, 27-35

45 Jongejan, F. (1991) The tick-borne rickettsia Cowdria ruminantium has a Chlamydia-like life cycle. Onderstepoort J. Vet. Res. 54, 197-204

46 Mahan, S. et al. (2001) Reduction of mortality from heartwater in cattle, sheep and goats exposed to field challenge using an inactivated vaccine. Vet. Parasitol. 97, 295-308

47 Jongejan, F. (1991) Protective immunity to heartwater (Cowdria ruminantium infection) is acquired after vaccination with in vitro attenuated rickettsiae. Infect. Immun. 59, 729-731

48 Jongejan, F. et al. (1993) Vaccination aginst heartwater using in vitro attenuated Cowdria ruminantium organisms. Rev. Elev. Med. Vet. Pays Trop. 46, 223-227

49 Gueye, A. et al. (1994) Essai sur le terrain d'un vaccin atténué contre la cowdriose. Rev. Elev. Med. Vet. Pays Trop. 47, 401-404

50 Zweygarth, E. and Josemans, A. (2001) Continuous in vitro propagation of Cowdria ruminantium (Welgevonden stock) in a canine macrophagemonocyte cell line. Onderstepoort J. Vet. Res. 68, 155-157

51 Collins, N. et al. (2003) Development of improved attenuated and nucleic acid vaccines for heartwater. Dev. Biol. (Basel) 114, 121-136

52 Zweygarth, E. et al. (2005) An attenuated Ehrlichia ruminantium (Welgevonden stock) vaccine protects small ruminants against virulent heartwater challenge. Vaccine 23, 1695-1702

53 Totté, P. et al. (1999) Immune responses to Cowdria ruminantium infections. Parasitol. Today 15, 286-290

54 DuPlessis, J.L. (1984) Colostrum-derived antibodies to Cowdria ruminantium in the serum of calves and lambs. Onderstepoort $J$. Vet. Res. 51, 275-276

55 Mwangi, D. et al. (2002) Immunization of cattle against heartwater by infection with Cowdria ruminantium elicits $\mathrm{T}$ lymphocytes that recognise major antigenic proteins 1 and 2 of the agent. Vet. Immunol. Immunopathol 85, 23-32

$56 \mathrm{Du}$ Plessis, J.L. et al. (1989) The heterogenicity of Cowdria ruminantium stocks: cross-immunity and serology in sheep and pathogenicity in mice. Onderstepoort J. Vet. Res. 56, 195-201 